\title{
Research on the Path of Improving Resource Integration Capability of Enterprise Based on QCA
}

\author{
QIU Yuxia \\ School of Management science and Engineering \\ Shanxi University of Finance and Economics, SXUFE \\ Taiyuan, China \\ tyqyxlj1@sina.cn
}

\author{
WU Tong* \\ School of Management science and Engineering \\ Shanxi University of Finance and Economics, SXUFE \\ Taiyuan, China \\ 1024460203@qq.com
}

\begin{abstract}
The cooperative relationship between enterprises brings convenience for them to integrate resources. However, based on different combinations of network structure and governance mechanism, resource integration capability of enterprise is different. In order to acquire central conditions, and matching rules between variables, this article according to resource based view and ability view constructed a theory model under the perspective of network governance. A questionnaire survey was carried out on the node enterprises of Linfen economic and Technological Development Zone, TISCO Stainless Steel Industrial Park and Houma Economic Development Zone and so on. QCA method was used to qualitatively compare and analyze 118 valid sample data. This study draws some conclusions that: Core conditions are trust mechanism and reputation mechanism. There is a matching relationship specific matching rule between the governance mechanism and the network structure. A key conditional combination influences the result most. Finally, the paper put forward corresponding suggestions to improve resource integration capability of enterprise with various structure types of network organizations. This research originally used QCA method to study a network governance problem due to its advantage on exploring the effect of combination on the result. It will have important reference value to improve resource integration capability, then improve network utilization efficiency and even promote enterprise performance in the end.
\end{abstract}

Keywords-Resource integration capability; Network structure; Network governance mechanism; Qualitative comparative analysis; Path

\section{INTRODUCTION}

With the development of economies and technologies, more and more scholars have paid close attention to resource integration problem. The ability to integrate resources is that according to the task, companies integrate its internal and external resources to generate new resources, and then allocate all of them to the production activities. In the process, it controls, motivates and coordinates group activities to make them integrate and create value for enterprises.[1].

Network relationship can bring rental benefits to the enterprise, and thus gradually become an important source of competitive advantage for the enterprises[2]. The resource integration through social network can improve the information transmission, reduce the risk caused by uncertain information, and improve the resources integration ability of enterprise[3].

Supported by the General project of Humanities and social sciences of Ministry of Education. (14YJA630047; 16YJA630047; 16BJY058)
Resource integration determines the success of technology innovation, and social network will promote the realization of technology innovation in enterprise.

\section{CONCEPTUAL MODEL}

As one of targets of network organization governance, improving resource integration capability is very significant for enterprises. But empirical researches for it is still confined within correlation analysis and no one have made researches on its improving mechanism, since the network structure elements and governance mechanism elements are basic elements of network organization. Therefore, investigating the impact of governance mechanism characteristics on the results of resource integration under diverse network structure characteristics will reveal the internal mechanism of it. Network structure refers to the form and intensity of the connection between actors, and different network forms and strength will produce different structures[4]. For the network structure factor, predecessors mainly focused on these three variables: the centrality of the network, strength of association and formal degree of agreement. The strength of association is the measurement of whether the connection is close and the frequency is high between members in the network organization. The formal degree of agreement refers to the degree of formality and strict compliance of contracts or agreements made by enterprises and cooperative enterprises before they cooperate. Network enterprises realize that central enterprises are more trustworthy and have higher status than non-central enterprises. They can also shorten the path length between central and non-central businesses in the network directly or indirectly, so that they can get information and resources more efficiently.[5][6].

Governance mechanism is the foundation of network organization's orderly operation. It directly affects the full play of the synergistic effect and the results of network governance[7][8].Based on the previous researches, we would utilize four variables to measure the governance mechanism: trust, reputation, joint sanctions, cooperation culture, and they would be analyzed in combination with the characteristics of the network structure. According to the definition of resource integration ability, the result would be measured by coordination ability and integration ability. Trust is a kind of psychological expectation and behavior expression that collaborator himself or the other side firmly believes that the 
other party will not sacrifice their interests to gain selfish interests[9]. It is a kind of abstract system, playing a basic regulating effect on the behavior of the network members[10]. To some extent, trust and close cooperation can reduce the cost of coordinated governance[11].

Reputation mechanism refers to the close cooperation relationship in the network, which makes the information more likely to convey to each member. In order to prevent losses caused by bad reputation, members will regulate their behavior. Joint sanction refers to collective punishments like public rumor, private discussion, deliberate vandalism and short-term deportation imposed on all member enterprises in the network organization that violate the norms of commonality. Cooperative culture mechanism is a system of the common values, behavior norms and expectations formed by industry, occupation and professional knowledge.

Therefore, this paper takes the structural factors and mechanism factors as the causal variables and resource integration ability as result variable to establish a conceptual model, such as Fig. I

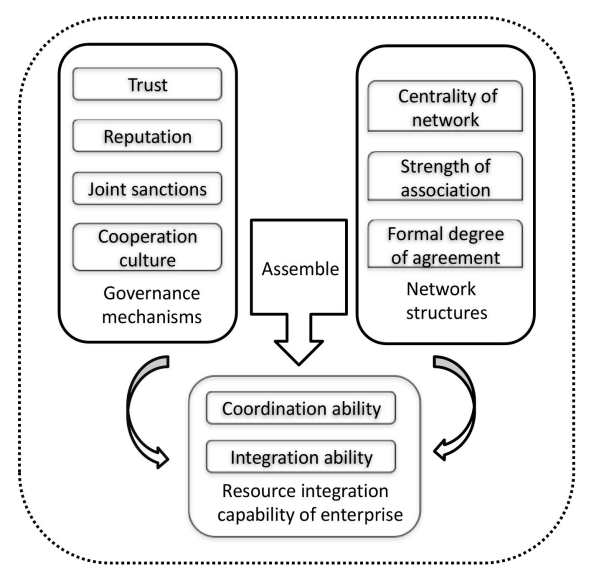

Fig. 1. Conceptual Model for Enhancing Resource Integration Capability

\section{VARIABLE MEASUREMENT AND DATA SOURCES}

We used a questionnaire survey to collect data. Firstly, on the basis of former researches and discussion in group, we formed the questionnaire using Likert 5 scale to weigh each item. Secondly, we invited 5 entrepreneurs to discuss and try filling in the questionnaire then modified the questions whose meaning was not clear or ambiguous in questionnaire; Finally, we gave out the questionnaires which including four part namely the basic situation of the enterprise, network structure, governance mechanism, and resource integration ability of enterprise through means of e-mail and interviews.

Data came mainly from the nodes enterprise in the three clusters which have typical network features, namely, Linfen economic and Technological Development Zone, TISCO Stainless Steel Industrial Park, and Houma Economic Development Zone. In order to make the questionnaire more accurately reflect the real situation of enterprises, the high and middle level leaders have been selected as the subjects to fill in the questionnaire. From July 20, 2015 to August 7th, we gave out 150 questionnaires in total, 134 questionnaires were received and the response rate was $89.300 \%$. Through further analysis and collation, we excluded 16 invalid questionnaires and finally 118 valid samples were left to analyze.

The reliability and validity of the questionnaire were tested by SPSS18.0, and results were shown in Table I. The reliability coefficient is 0.875 , so, result is reliable. The Bartlett sphericity test was performed on the valid sample data, and the value of Bartlett sphericity test was 282.689 , reaching a significant level $(p=0.000<0.001)$. The value of KMO test is 0.814 . When KMO is greater than 0.600 , we can carry on factor analysis, so, the sample data from questionnaire is suitable for factor analysis. Then, the principal component analysis was applied to Varimax variance orthogonal rotation, and finally, 4 factors with eigenvalues greater than 1 were extracted. The cumulative variance contribution rate was $59.326 \%$, indicating that the validity of the questionnaire was good.

TABLE I. RELIABILITY AND VALIDITY OF QUESTIONNAIRE

\begin{tabular}{|c|c|c|c|}
\hline Indexes & Statistics & Indexes & Statistics \\
\hline $\mathrm{N}$ & 118 & KMO & 0.814 \\
\hline Cronbach's & 0.875 & Bartlett's checkout & 282.689 \\
\hline $\begin{array}{c}\text { Cumulative explanatory } \\
\text { variance/\% }\end{array}$ & $59.326 \%$ & df & 28.000 \\
\hline & & Sig. & 0.000 \\
\hline
\end{tabular}

\section{QUALITATIVE COMPARATIVE ANALYSIS}

Qualitative comparative analysis is proposed by Ragin in 1987[12]. It can effectively handle and compare multi-case study data to find out the causes of the results and the interaction between the generation factors, which is an analysis method that takes the case study and the causes of the results as the reasons. The reasons why this paper to use this method are as follows:

The advantage of this method is to analyze small sample data. Therefore, it can analyze the cause conditions of result indepth in order to find out a variety of ways and channels and measure the net influence of different reasons on the result[13]. Cases used in this paper are 118 , but there are 8 variables in this paper, the number of samples is relatively small.

The object of this paper belongs to the problem which need empirical causal reasoning but cannot be deducted.

This method focused on the necessary and sufficient conditions for a given result, and it is not easily affected by the unfavorable effect caused by multicollinearity and autocorrelation.

QCA is suitable for analyzing high level stochastic complexity[14].

Based on the binary calibration method, the average value of the variable are the calibration lines. When values higher than the calibration line, the assignment are 1, otherwise, it is 0 . In the software, when "non" was pulsed before the variable name, it indicates the variable value is 0 , otherwise, it is 1 .

This paper uses version 2.5 of fsQCA software to complete all the following data analysis. 


\section{A. Necessary Condition Analysis}

The results of necessary conditions analysis are shown in Table II. The $p$ value in QCA method refers to the degree of consistency between the variables and the result, and it describes extent a result requires the presence of a variable or a variable combination. When the consistency degree reached 0.900 and the variables covered $90 \%$ of the cases, we can consider the condition variable is a necessary condition for the occurrence of result[15].

TABLE II. The Necessity Test of CONDItional VARIABles

\begin{tabular}{|c|c|}
\hline Variables & Consistency \\
\hline Trust & 0.833 \\
\hline Reputation & 1.000 \\
\hline Joint sanctions & 0.650 \\
\hline Cooperation culture & 0.467 \\
\hline Centrality of network & 0.450 \\
\hline Formal degree of agreement & 0.850 \\
\hline Strength of association & 0.533 \\
\hline
\end{tabular}

All the 7 condition variables are listed in Table II . The necessary condition is reputation mechanism with consistency reached 1. It indicates that the occur of reputation mechanism often cause high ability to integrate resources; Variables which close to be necessary is trust mechanism and formal degree of agreement. The consistencies of them were 0.833 and 0.850 . It indicates these two variables have great influences on result variable, but they are not necessary conditions of result. We need to analyze the combination of all this conditions in addition to reputation mechanism.

\section{B. Conditional Combination Analysis}

According to the above results, we input all the condition variables and result variable in the QCA software except reputation mechanism. We set the frequency threshold be 2 , because the case occurred for just 1 time cannot eliminate the opportunity of accidence. The consistency threshold is 0.800 , because when the consistency index is greater than 0.800 , it indicates there are eighty percent cases in accordance with the conditions, which can be considered that the path is the necessary condition of result arising. Running results by software are shown in table III:

TABLE III. RUNNING RESUlTS OF QCA SOFTWARE

\begin{tabular}{|c|c|c|c|c|}
\hline \multirow{2}{*}{ Conditions } & \multicolumn{4}{|c|}{ Paths } \\
\hline & Path1 & Path2 & Path3 & Path4 \\
\hline Centrality of network & 0 & - & $\otimes$ & $\otimes$ \\
\hline Formal degree of agreement & O & $\otimes$ & 0 & 0 \\
\hline Strength of association & & & $\otimes$ & 8 \\
\hline Trust & 0 & & 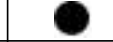 & \\
\hline Joint sanctions & $\otimes$ & 0 & e & 0 \\
\hline Cooperation culture & $\otimes$ & 0 & $\otimes$ & a \\
\hline Consistency & 1.000 & 1.000 & 1.000 & 1.000 \\
\hline Raw coverage & 0.083 & 0.067 & 0.067 & 0.217 \\
\hline Unique coverage & 0.083 & 0.067 & 0.067 & 0.217 \\
\hline
\end{tabular}

\begin{tabular}{|l|l|}
\hline \multicolumn{2}{|c|}{ Cont. to TABLE III } \\
\hline Solution consistency & 1.000 \\
\hline Solution coverage & 0.433 \\
\hline
\end{tabular}
Notes: Black circle means the condition exists; while circle means the condition does not exist; "Blank" said the condition exist or not doesn't affect the result; Big circle implies that it is the core condition condition which have a weak causal relationship with the result.

\section{The result shows that:}

Trust mechanism is the core condition. Trust relationship as an "adhesive" is the foundation of forming network organization; meanwhile it is an indispensable power of successful operation and development of network organization. It can encourage enterprises to adopt cooperative behavior, reduce uncertainty, reduce the opportunism behavior, and improve coordination between enterprises[16]. There are four paths to obtain high resource integration ability, of which path4 is the critical path. Its net coverage rate is about 0.217 , which is much higher than the other three paths $(0.083,0.067,0.067)$. The highest importance of this path implies that all the cases that have gotten high ability mostly enjoy characteristics such as low centrality of network, high strength of association and high formal degree of agreement and sound governance mechanism. There are some matching relationship between the governance mechanism and the network structure. Firstly, strength of association and cooperation culture mechanism is complementary (path3,4). Under the cross-cultural background, enterprises may have cultural differences caused by different country, different types and different management styles, which easily lead to poor communication and even conflict; Secondly, centrality of network and joint sanctions mechanism have an alternative relationship (path1,3), namely the network center is located at the high level, and the existence of the joint sanctions mechanism is not important. Because in this situation, enterprise occupy a position which is powerful, it can restrain or punish others for violating the rules[17]. By contrary, when the centrality of network is low, joint sanctions mechanism must be exist, and it could be better executed; Thirdly, when the centrality of network is high, low or high strength of association both can achieve the same effect (path1,4). When the network has a central enterprise, the central enterprise through collecting and processing intermediary information can improve the exchange of information between enterprises. Thus, appropriate matching between different network structures and governance mechanisms can give enterprise a high ability to integrate resources.

\section{CONCLUSION AND DISCUSSION}

\section{A. Conclusion}

In order to explore specific path and key elements to enhance resource integration ability of enterprise in the network, this paper takes the result as the ability of interaction of the network structure and governance mechanism. So, firstly, we built a conceptual model to promote the ability based on network governance; Secondly, according to the actual requirements by using QCA method, we studied the paths and methods to make it. Finally, we obtained the results which are shown in table IV: 
TABLE IV. FINDINGS

\begin{tabular}{|c|l|}
\hline & \\
\hline Core conditions & Trust mechanism, Reputation mechanism \\
\hline Key path & $\begin{array}{l}\text { High formal degree of agreement *High strength of association*Low centrality of network *Trust mechanism*Cooperation culture } \\
\text { mechanism*Joint sanctions mechanism }\end{array}$ \\
\hline Matching rules & $\begin{array}{l}\text { Strength of association complementary with cooperation culture mechanism; Centrality of network substitute for joint sanctions } \\
\text { mechanism; Centrality of network complementary with strength of association }\end{array}$ \\
\hline Note: "*" means "and"
\end{tabular}

\section{B. Discussion}

The network organization with characteristics like low centrality of network, high formal degree of agreement and high strength of association is called peer network. We should perfect the governance mechanism; the network organization with characteristics like high centrality of network and high formal degree of agreement is called scale-free networks. We should establish trust mechanism; the network organization with characteristics like low centrality of network, high formal degree of agreement and low strength of association is called temporary cooperative network we should establish trust mechanism and joint sanctions mechanism.

\section{REFERENCES}

[1] Koruna S. Leveraging knowledge assets: combinative capabilities: a review, model and research agenda [J]. Journal of Management Studies, 2006, 43 (4): 917-955.

[2] Dyer J, Singh J. The relational view: cooperative strategy and sources of inter-organizational competitive advantage[J]. Academy of Management Review, 1998,23(4):660-679.

[3] Wu Shaoyu, Wang Dong, Wang Bo, Li Xiaoyan. Research on the effect path of entrepreneurial social networks on re entrepreneurial performance [J]. Journal of Science Research, 2016, (11): 1680-1688.

[4] Myerson. Graphs and cooperation in gamer[J].Mathematics of Operations Research,1997,2(3):225-229.

[5] Ibarra H. Network centrality, power, and innovation involvement: determinants of technical and administrative roles[J]. Academy of Management Journal, 1993,36(3), 471-501.

[6] Perry - Smith J E. Social yet creative: the role of social relationships in facilitating individual creativity $[\mathrm{J}]$. Academy of Management Journal, 2006,49(1),85-101.
[7] Mi Ya. Review of industrial cluster governance [J]. Journal of Modern Economic Information, 2014 (17): 113-113.

[8] Han Wei, Yang Wanyu. Research on the relationship between entrepreneurial network governance mechanism, network structure and new enterprise performance $[\mathrm{J}]$. Journal of Management Review, 2015,27 (12): 65-79.

[9] Sun Guoqiang. Research on governance mechanism of network organization[D]. Tian Jin: Nankai University, 2004:86-97.

[10] Sun Guoqiang. Governance mechanism of network organization[J].Organization Behavior, 2003, (4): 39-41.

[11] Uzzi B.Social structure and competition in inter-firm networks: the paradox of embeddedness[J].Administrative Science Quarterly,1997,4(2):35-67.

[12] Lin H,Lin C,Huang H. Embedding Strategic Alliances in Networks to Govern Transaction Hazards: Evidence from an Emerging Economy [J].Asian Business \&Management,2011,10(2):183-208.

[13] Wang Fengbin, Zheng Xiaojie, Chen Gonghai, Wang Cong. Management elements linkage effects and central enterprise management to enhance - A cross-layer comparison analysis based on management system network characteristics[J].Journal of China's Industrial Economy, 2014.5 (5): 135-147.

[14] Rihoux B, Ragin C C. Configurationally comparative methods: Qualitative comparative analysis(QCA) and related techniques[J].Thousand Oaks, CA: Sage,2009,6(9):89-106.

[15] Bao Xinzhong, Tao Qiuyan, Sheng Xiaojuan. An empirical study of the impact of post-merger integration on Innovation: an analysis based on the synergy effects of resource integration [J]. Journal of East China Economic Management, 2014,28 (8): 101-106.

[16] Zhao Yanzhi, Zhou Shouliang. The relationship between network governance and innovation performance of scientific research organizations from the perspective of multiple Embeddedness[J]. Journal of Economic Management, 2016, (12): 170-182.

[17] Mehra A, Dixon A L, Brass D J, Robertson B. The social network ties of group leaders: implications for group performance and leader reputation[J].Organization Science,2006,17(1),64-7. 